\title{
BACK-EMF BASED ROTOR POSITION ESTIMATION FOR LOW COST PMSM DRIVE USING FULLY CONNECTED CASCADE ARTIFICIAL NEURAL NETWORKS
}

\author{
Gabriel H. Negri ${ }^{1}$, Filipe G. Nazário ${ }^{1,2}$, José de Oliveira $^{1}$, Ademir Nied ${ }^{1}$ \\ ${ }^{1}$ Santa Catarina State University, Joinville - SC, Brazil \\ ${ }^{2}$ EMBRACO, Joinville - SC, Brazil \\ e-mail: \{negri.gabriel, filipef2\}@gmail.com, \{ademir.nied, jose.oliveira\}@udesc.br
}

\begin{abstract}
Permanent Magnet Synchronous Motors (PMSMs) are widely used mainly due to their high torque per volume, high efficiency and low maintenance cost, among other advantages. To perform vector control on rotor speed and stator currents, the feedback of those variables is necessary, which can be done directly or by estimation. Measuring rotor position and speed directly requires the use of a mechanical device attached to the motor shaft, increasing the drive system volume and its maintenance cost. To overcome such disadvantages, many sensorless methods for speed estimation have been proposed. Among those methods, various strategies based on Artificial Neural Networks (ANNs) can be found. This paper presents a back-electromotive force estimator based on Fully Connected Cascade ANNs (FCC-ANNs). From the estimator, rotor position and speed can be obtained. Simulation and experimental results using automatically generated $\mathrm{C}$ code functions for the FCC-ANNs using fixed point notation provided rotor position estimation with simple implementation.
\end{abstract}

Keywords - Artificial Neural Networks, Back-EMF Estimation, Permanent Magnet Synchronous Motor, Position Estimation.

\section{NOMENCLATURE}

$\begin{array}{ll}v_{\alpha, \beta} & \alpha \text { and } \beta \text { axis voltages. } \\ v_{a, b, c} & a, b \text { and } c \text { phase voltages. } \\ i_{\alpha, \beta} & \alpha \text { and } \beta \text { axis currents. } \\ i_{a, b, c} & a, b \text { and } c \text { phase currents. } \\ E_{\alpha, \beta} & \alpha \text { and } \beta \text { axis back-EMFs. } \\ E_{a, b, c} & a, b \text { and } c \text { phase back-EMFs. } \\ \omega_{r} & \text { Rotor electric angle frequency. } \\ \omega_{m} & \text { Rotor mechanical angle frequency. } \\ \theta_{r} & \text { Rotor electric angle position. } \\ L & \text { Phase inductance. } \\ T_{L} & \text { Load torque. } \\ L_{M} & \text { Phase mutual inductance. } \\ T_{e} & \text { Eletromagnetic torque. } \\ R & \text { Phase resistance. } \\ P & \text { Number of poles. } \\ h & \text { Viscous friction coefficient. } \\ J & \text { Total inertia. } \\ k_{e, t} & \text { Back-EMF and torque coefficients. }\end{array}$

Manuscript received 17/04/2017; first revision 06/06/2017; accepted for publication 19/08/2017, by recommendation of Editor Marcelo Cabral Cavalcanti.

\section{INTRODUCTION}

Permanent Magnet Synchronous Motors (PMSMs) are widely employed due to their advantageous features, comparing with other types of motors. Among such features, the reduced volume, low maintenance cost and high efficiency can be mentioned. PMSMs are employed in systems where high dynamic performance is required, such as robotics, and for energy saving in home appliances, automotive applications, among others, due to their high efficiency [1]. To perform current and speed control, information of rotor position and voltage and current measurements are necessary. Such information can be obtained directly or indirectly. The use of mechanical devices such as encoders or resolvers enables the direct position feedback, but causes an increase in the drive system volume, in addition to increasing maintenance and manufacturing costs [2]. PMSMs are usually divided into sinusoidal PMSM, in which the magnets are distributed in a manner that the back-EMFs have sinusoidal waveforms and non-sinusoidal PMSM, in which the backEMFs present trapezoidal waveforms [3].

Sensorless techniques aim to eliminate the need of mechanical sensors, reducing the drive maintenance and acquisition cost. For a sensorless method to be accepted in industry, it has to fulfill some requirements. Among such requirements, it must not increase the drive cost with the need of more powerfull and expensive computational technology [4].

In the indirect form, many methods can be used to estimate the motor electric angle position and speed, such as modelreference adaptive system (MRAS), sliding mode observer (SMO) and estimators based on artificial neural networks (ANNs) [2], [5], [6]. The main advantages in using ANNs are the nonlinear input-output mapping achieved through training and the generalization characteristic, allowing the ANN to predict the motor dynamics in different conditions, using only sample data.

Other types of nonlinear mapping involve lookup tables and support vector regression. Such methods, however, have a larger computational burden than feedforward ANNs [6]. Many applications of sensorless control to electric machinery can be found, mainly for induction motors (IMs). For IM drive systems, ANNs can be used to estimate rotor resistance and/or rotor speed, rotor flux and obtaining the electrical position based on such variables [7], [8]. Other strategies for IM drives can be found, such as the use of a Luenberger observer and MRAS strategy for Direct Torque Control (DTC) [9].

As for the use of ANNs as state estimators, several works 
can be found in literature. One example is the application diagonal recurrent neural networks, used to compose the observer [2]. In such work, two neural observers and a position estimation correction block are used. One neural observer is used to estimate stator current and the second observer is used to estimate speed from measured stator currents and voltages. The estimated rotor speed is then integrated for obtaining rotor position.

A review on sensorless control techniques for nonsinusoidal PMSM (the Brushless DC motor) drive can be found in [5]. Among the presented estimation techniques, the ANN approach is mentioned. The authors comment on the ANN capability for approximating nonlinear functions and cite some applications in motor drives, such as online parameter (resistance and inductance) and torque estimation in vector-controlled induction motor drives, which can be also applied in PMSM drives.

Another ANN-based rotor position estimation, for a $8 / 6$ solid rotor switched reluctance motor, is presented in [10]. Such type of motor is utilized mainly due to its robustness and fault tolerance. ANNs are used to estimate rotor position from phase flux linkage and phase current input data. The utilized drive consists in four different operating regions. Thus, four ANNs are utilized, one for each operating region. The authors comment on an important characteristic of the ANN approach, which is the automatic consideration of nonlinear effects, such as mutual inductance and eddy currents effects, in the inputoutput mapping.

In addition to acting as observers, ANNs have also been used within the control algorithm for nonlinear control of eletric machinery. In [11], an ANN was combined with a fuzzy logic to develop a speed controller for a non-sinusoidal PMSM. The obtained dynamic response was compared to other controllers, such as the conventional proportionalintegral and other fuzzy strategies. The authors commented that the proposed ANN-fuzzy controller outperformed the others in terms of dynamic characteristics, as settling time and overshooting. Another model-based ANN control approach using ANN is presented in [12]. Two ANNs are used in the control loop of a DC motor drive, in which one network is used to estimate the drive dynamics and the other is used to generate voltage input signals to the drive. Different applications of ANNs in engineering include system identification, fault detection and power electronics applications, among others [13]-[15].

Although methods used in previous works present satisfactory results, the objective of this paper is to present a simpler ANN-based observer, providing the used training data, simulation and experimental results, without using online training. The main idea of the work presented here is to estimate rotor position for then obtaining the speed estimation, in a manner that the estimator has low computational cost and be of easy implementation, exploiting the advantages of ANNs mentioned in the literature to obtain a simple, fast and straightforward estimator. Without using integration of estimated speed to obtain the electrical position, the position correction block is eliminated. Based on a Luenberger observer, which takes the voltages and currents in the $\alpha \beta$ reference-frame (stationary) to estimate the $\alpha$ and $\beta$ back-electromotive forces (back-EMFs), the use of an ANN for estimate each back-EMF is proposed [16]. Using such estimations, it is possible to obtain the estimated rotor angle position. After training the networks, $\mathrm{C}$ code functions using fixed point notation with integer bases are automatically generated and the implementation consists in uploading such functions to the microcontroller. Thus, the ANNs are coded as black-box functions, which take as inputs the measured stator currents and applied voltages and return the estimated back-EMFs. Another objective of this work is to present the application of Fully Connected Cascade (FCC) ANNs, which can achieve, with fewer hidden neurons, the same performance of a typical Multi Layer Perceptron (MLP) network, thus reducing the computational burden.

The procedure for training the ANN is to apply several speed reference steps, using an encoder to measure rotor position, and organizing the acquired data to train the ANNs. With the trained ANNs, the encoder can be replaced by the ANN-based estimator. It is interesting for a sensorless method that parameter tuning be as less manual as possible, reducing instalation time [4]. The proposed estimation method in this work has this advantage. Once the ANN architecture and a minimal number of neurons is defined, the procedure for training the ANN-based estimator for other different PMSM is similar. As mentioned before, another advantage of an ANN approach is the automatic treatment of the drive nonlinearities. Thus, it is possible to highlight the main features of the presented observer as:

- Use of FCC topology, reducing the number of connections and computational burden if compared to classical MLP networks [17];

- The PMSM parameters do not need to be identified individually;

- The position estimation does not depend directly on the signals amplitude, since it is obtained based on the position of the estimated back-EMFs in $\alpha \beta$ notation, which is useful for dealing with load disturbances and nonlinearities;

- C code functions using fixed point notation are automatically generated after training, which simplifies implementation on a low cost microcontroller (see Figure 27 in Appendix section for an example of automatically generated code).

The use of an encoder in the training process is necessary since the alternative would be to utilize another estimator to generate the data for training, which would require an entire tuning process. Additionally, in industry applications, the encoder is also utilized for tuning other types of observer, because it is necessary not only to identify model parameters but also to validate the results from the observer with a reliable sensor. Thus, the use of the encoder in training process of the method described in the present paper does not represent a drawback in comparison to other observer methods applied industrially.

In Section II, the dynamic model for the PMSM is presented. The design and implementation aspects of the estimator are presented in Section III. Simulation results are 
shown in Section IV the experimental results in Section V. Conclusions are discussed in Section VI.

\section{SINUSOIDAL PMSM DYNAMICS}

The electrical dynamics for a balanced three-phase wyeconnected sinusoidal PMSM can be writen by terms of phase variables as [18]:

$$
\begin{aligned}
& v_{a}=R i_{a}+\left(L-L_{m}\right) \frac{d i_{a}}{d t}+E_{a} \\
& v_{b}=R i_{b}+\left(L-L_{m}\right) \frac{d i_{b}}{d t}+E_{b} \\
& v_{c}=R i_{c}+\left(L-L_{m}\right) \frac{d i_{c}}{d t}+E_{c}
\end{aligned}
$$

where $E_{a}, E_{b}$ and $E_{c}$ are the phase back-EMFs, given by:

$$
\begin{gathered}
E_{a}=k_{e} \omega_{r} \sin \left(\theta_{r}\right) \\
E_{b}=k_{e} \omega_{r} \sin \left(\theta_{r}-120^{\circ}\right) \\
E_{c}=k_{e} \omega_{r} \sin \left(\theta_{r}+120^{\circ}\right)
\end{gathered}
$$

in which $\omega_{r}$ is the rotor electrical speed and $\theta_{r}$ is the rotor electrical position.

The conversion of a set of balanced three-phase variables $f_{a}, f_{b}$ and $f_{c}$ to two-phase variables $f_{\alpha}$ and $f_{\beta}$ is made using Concordia's Transform [19], to eliminate lineary dependent variables:

$$
\left[\begin{array}{l}
f_{\alpha} \\
f_{\beta} \\
f_{0}
\end{array}\right]=\frac{2}{3}\left[\begin{array}{ccc}
1 & -\frac{1}{2} & -\frac{1}{2} \\
0 & \frac{\sqrt{3}}{2} & -\frac{\sqrt{3}}{2} \\
\frac{1}{2} & \frac{1}{2} & \frac{1}{2}
\end{array}\right]\left[\begin{array}{l}
f_{a} \\
f_{b} \\
f_{c}
\end{array}\right]
$$

The opposite process is made through the inverse of the transformation matrix utilized in (7).

Considering the $\alpha \beta$ reference-frame and using (7), the following equations can obtained to represent the electrical dynamics of the PMSM:

$$
\begin{aligned}
& v_{\alpha}=R i_{\alpha}+\left(L-L_{m}\right) \frac{d i_{\alpha}}{d t}+E_{\alpha} \\
& v_{\beta}=R i_{\beta}+\left(L-L_{m}\right) \frac{d i_{\beta}}{d t}+E_{\beta} .
\end{aligned}
$$

The produced torque can be written in terms of phase currents and back-EMFs as:

$$
T_{e}=\left(\frac{k t}{k e}\right)\left(\frac{P}{2}\right) \frac{E_{a} i_{a}+E_{b} i_{b}+E_{c} i_{c}}{\omega_{r}} .
$$

The mechanical dynamics are given by:

$$
J \frac{d \omega_{m}}{d t}=T_{e}-T_{L}-h \omega_{m}
$$

where $h$ is the friction coefficient of the mechanical coupling.

The relation between the electrical frequency and the mechanical frequency is given by:

$$
\omega_{r}=\frac{P}{2} \omega_{m} .
$$

\section{BACK-EMF ESTIMATOR USING FCC ARTIFICIAL NEURAL NETWORKS}

In this section, the used estimator based on FCC-ANNs is presented. Firstly, the estimation strategy is presented, indicating the role of the ANNs in such strategy. Then, the ANN architecture, topology and other parameters are commented.

\section{A. Estimation Strategy}

A flowchart for the proposed position and speed estimator is shown in Figure 1. The method consists in performing the back-EMFs estimation in the $\alpha \beta$ system to first obtain the position and then speed. The observer is composed by a pair of neural networks. Each network receives as inputs the voltages and currents $\alpha$ and $\beta$, and estimates each one of the backEMFs. The estimated back-EMFs are calculated by an arctangent algorithm. The angular frequency, namely speed, is then estimated by the position variation rate.

The derivative terms in (8) and (9) are not used in the training process. This was done because of the ANNs capability of estimating the back-EMFs without such terms, due to their memorization characteristics. Also, the derivative terms insert a high noise level in practice.

As the electrical dynamics of the motor is fast, compared to the mechanical dynamics, the information of lead/lag and relative amplitude between voltage and current are sufficient for the back-EMFs estimation. Information of phase and amplitude difference of the voltage and current signals due to inductances and resistances are implicitly learned by the network during the training. This way, one of the advantages of using neural networks is evident, which is the parameter independency. In other words, the estimation can be performed from experimental data without knowing the motor parameters, such as phase equivalent resistance and inductance.

\section{B. ANNs for the Estimator}

The utilized neural network architecture is the FCC-ANN, trained with Neuron by Neuron (NBN) algorithm [17]. Each hidden layer in a FCC-ANN has only one neuron. The output of each neuron is connected to all of the forward neurons. Such architecture was used for having better performance with a lower number of neurons and synaptic connections if compared to the traditional MLP, provided that the training is adequate [20]. The reduced number of neurons and connections contributes with the implementation in a microcontroller device, given the limited memory and processing performance of the device. The topology consist in 4 data inputs plus 1 bias input, 4 hidden neurons and

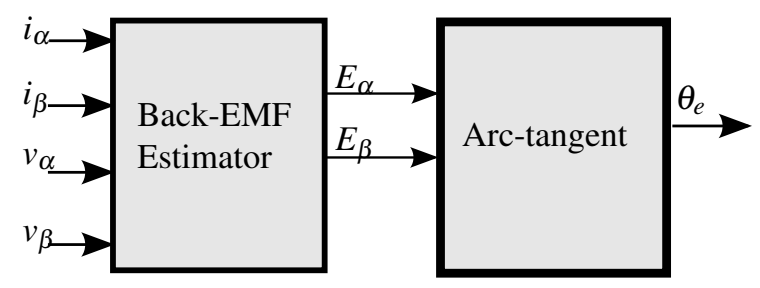

Fig. 1. Estimator flowchart. Based on [16]. 
one output neuron. The activation is the Symmetric Elliot's function, mainly for being of easy implementation in fixed point notation, given by:

$$
\operatorname{Elliot}(x)=\frac{2 a x}{1+|a x|} .
$$

In the simulations with floating point, the Symmetric Sigmoid function presented the best performance, but it takes approximations or iterative calculations to be implemented in the utilized hardware, which prevented its use. The parameter $a$ represents the function steepness and $x$ is the activation potential (inputs weighted sum with the synaptic weigths of the neuron inputs). The implementation was made using SuperNN library [21].

The training was performed using the measurements of the $\alpha$ and $\beta$ voltages and currents and rotor position and speed using an encoder. Such data was acquired with the mechanical speed varying from 30 RPM to 120 RPM. The back-EMFs signals for the training are obtained through the product of the sines and cosines of the position and the measured speed, taking the back-EMF constant unitary. The back-EMF coefficient value is not necessary once the position in given by an arctangent function which takes the ratio between the two back-EMFs. However, it is necessary to generate the back-EMFs amplitude variating with the speed for the network to be trained appropriately. The experimental training data set and the simulation training data set were assembled with approximately 3000 samples each.

Figure 2 shows the ANNs structure. Note that each of the ANNs produces only one output, $E_{\alpha}$ or $E_{\beta}$. Using only one ANN with two outputs highly increased the number of necessary neurons and connections to achieve a similar performance than with two separated ANNs.

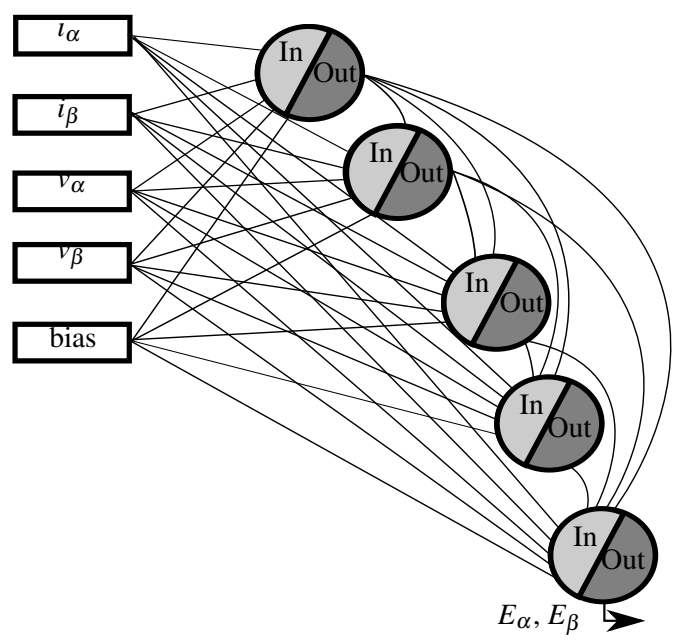

Fig. 2. ANNs FCC topology.

\section{SIMULATION RESULTS}

The motor parameters are given in Table I. It is a motor utilized in washing machines, with a high number of poles, in a manner that the operating mechanical rotation is relatively low comparing to the drive frequency.
TABLE I

Motor Parameters

\begin{tabular}{ccc}
\hline Parameter & Value & Unit \\
\hline$R$ & 15.5 & $\Omega$ \\
\hline$L$ & $76 \times 10^{-3}$ & $\mathrm{H}$ \\
\hline$L_{m}$ & $38 \times 10^{-3}$ & $\mathrm{H}$ \\
\hline$J$ & 0.1566 & $\mathrm{Kg} \times \mathrm{m}^{2}$ \\
\hline$\beta$ & $0.98 \times 10^{-3}$ & $\mathrm{~N} \times \mathrm{s} / \mathrm{m}$ \\
\hline$P$ & 48 & - \\
\hline$k_{e}$ & 0.117 & $\mathrm{~V} \times \mathrm{s} / \mathrm{rad}$ \\
\hline$k_{t}$ & 0.117 & $\mathrm{~N} \times \mathrm{m} / \mathrm{A}$ \\
\hline
\end{tabular}

The simulated data for training was obtained by controlling the simulated motor dynamics over several speed reference steps, with and without load. To consider the presence of noise in currents and voltages, to verify the estimator robustness, a random relative noise of $40 \%$ was added to the measured voltages and currents.

\section{A. Simulations without Load}

The back-EMFs estimation for the motor rotating at frequencies of 30 RPM, 50 RPM and 100 RPM are depicted in Figures 3, 4 and 5, respectively. In all three cases it can be observed that the $\alpha$ and $\beta$ estimated back-EMFs have a $90^{\circ}$ phase shift, with amplitude variations caused by the inserted noise in current and voltage. The amplitude and the frequency of the signals increase proportionally with the speed.

From the estimated back-EMFs, rotor electrical position can be obtained. Simulation results are shown in Figures 6, 7 and 8.

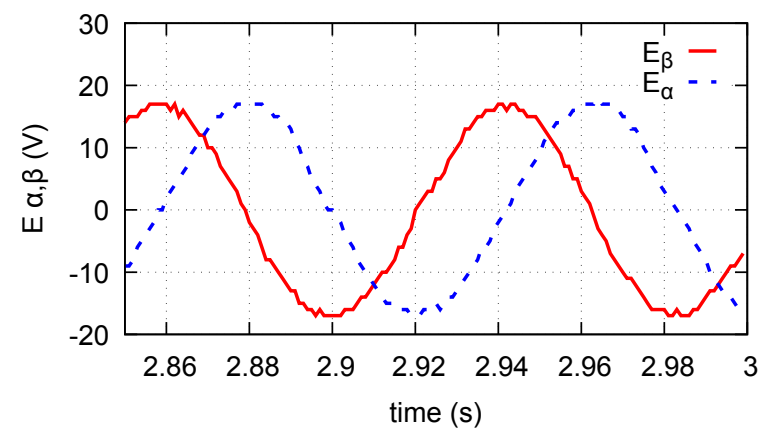

Fig. 3. Simulation: back-EMF estimation at 30 RPM.

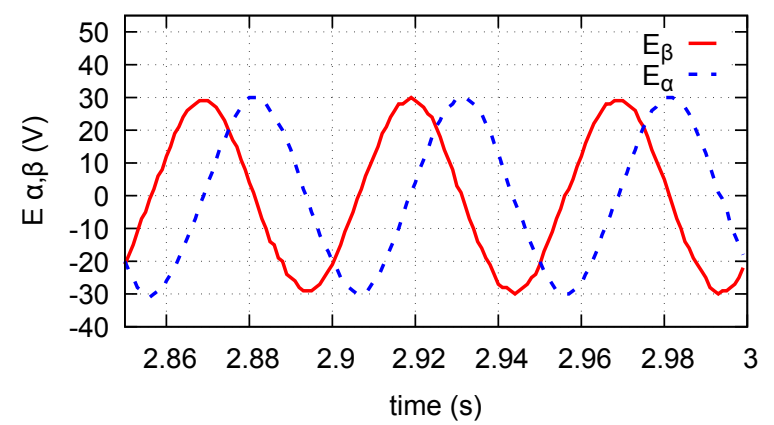

Fig. 4. Simulation: back-EMF estimation at 50 RPM.

\section{B. Simulations with Load}

Simulations with the application of a $2 \mathrm{Nm}$ load step, at $t=2.5 \mathrm{~s}$ were performed to evaluate the estimator under load 


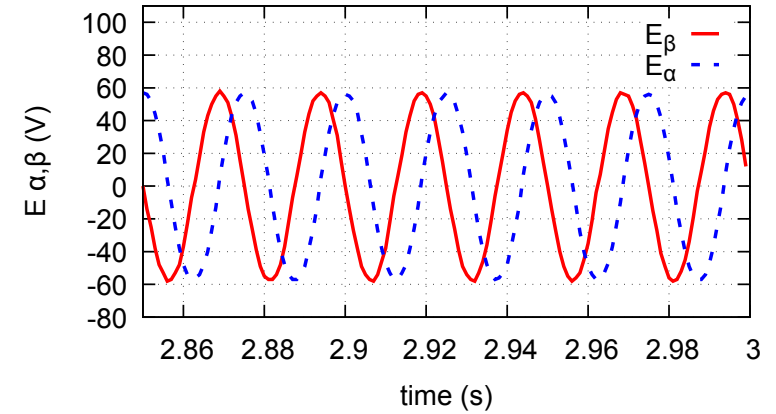

Fig. 5. Simulation: back-EMF estimation at 100 RPM.

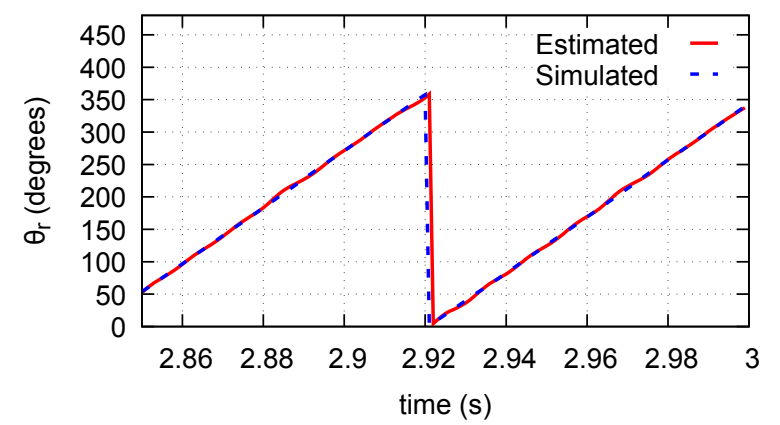

Fig. 6. Simulation: position estimation at 30 RPM.

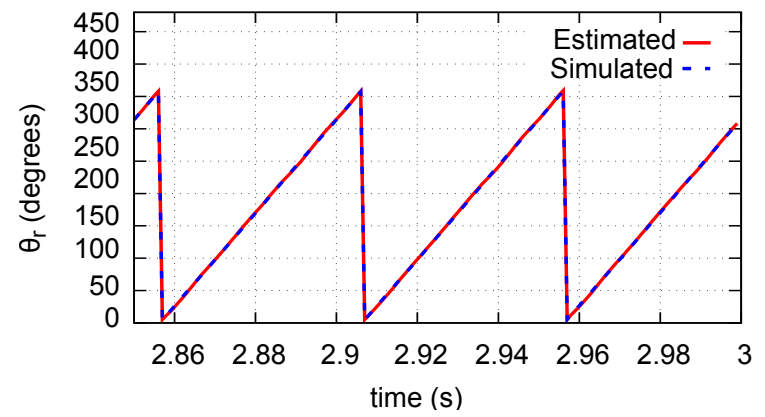

Fig. 7. Simulation: position estimation at 50 RPM.

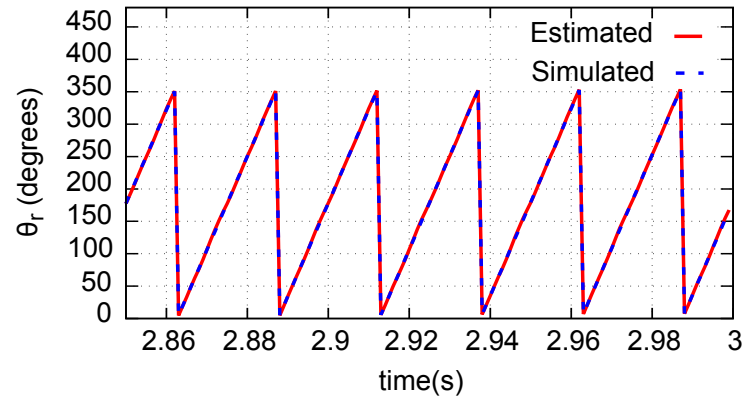

Fig. 8. Simulation: position estimation at 100 RPM.

disturbance. Figures 9,10 and 11 present the estimated and simulated positions at mechanical speeds of 30 RPM, 50 RPM and 100 RPM respectively. At the insertion of the load, it is possible to observe oscillations in the estimated position, which were stabilized with time. After stabilization, the estimations with load presented coherent behaviors with the tests without load. However, a higher noise can be observed at 30 RPM than in the test without load, since current increases with load and a proportional noise was used in simulation.

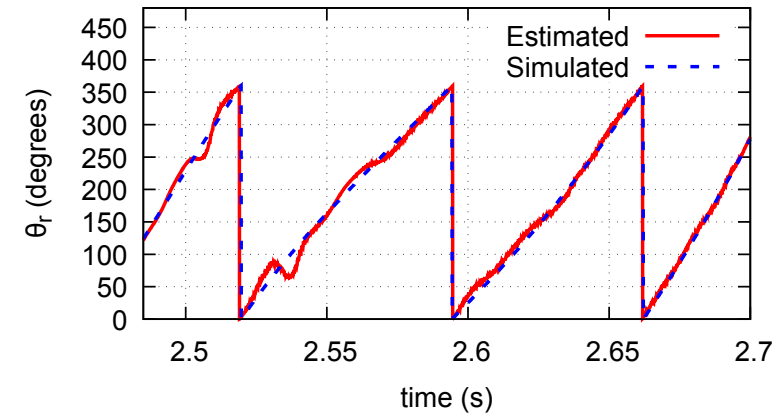

Fig. 9. Simulation: position estimation at 30 RPM with load.

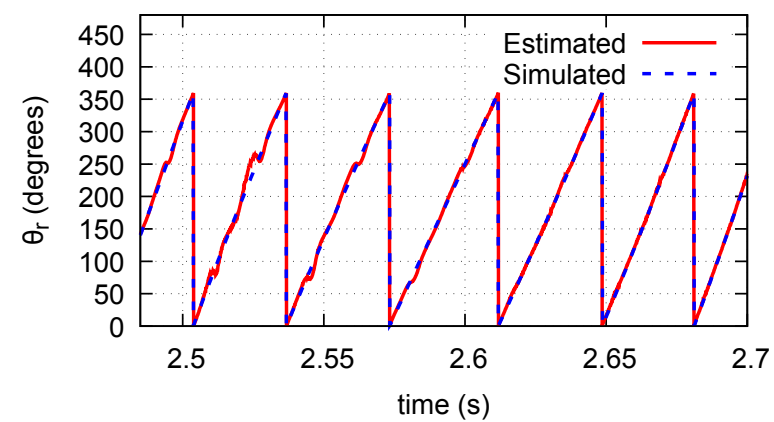

Fig. 10. Simulation: position estimation at 50 RPM with load.

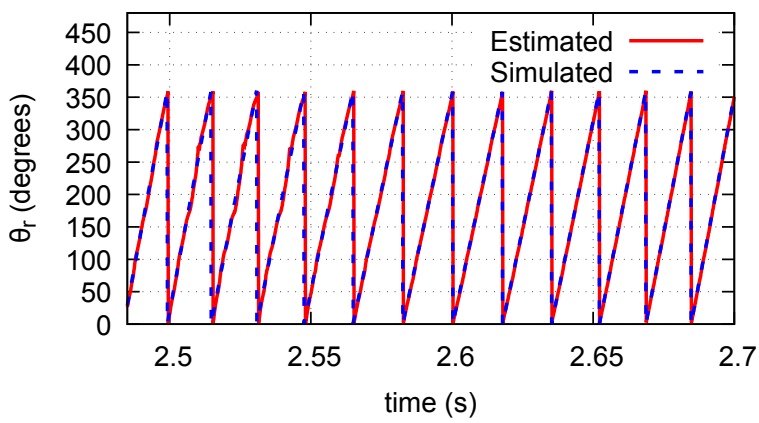

Fig. 11. Simulation: position estimation at 100 RPM with load.

\section{EXPERIMENTAL RESULTS}

Experimental results were obtained using a PMSM drive with vector control. The ANNs were executed along with the readings of an encoder, to verify the results. In this section, training procedures are first presented, followed by experimental results obtained in the execution phase.

\section{A. Training}

Several speed reference steps were applied to obtain a data set with information of the motor behavior from 30 RPM up to 120 RPM. Due to hardware limitations in the acquisition system, only four variables could be obtained simultaneously. The chosen variables are: $\beta$ voltage, $\beta$ current, electrical position and speed. The $\alpha$ variables are then obtained by shifting the $\beta$ variables by $90^{\circ}$. Figure 12 shows the assembled data set for voltages while Figure 13 shows the data set for currents. The value ranges are related to internal microcontroller variables. The ANN topology is the same as the mentioned in Section III, Subsection B, with 4 
hidden neurons. Training was performed through 300 epochs with NBN algorithm.

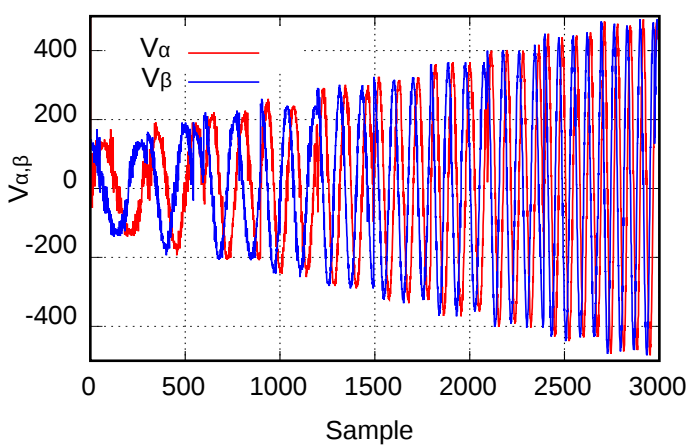

Fig. 12. Experimental $\alpha$ and $\beta$ voltage data for training.

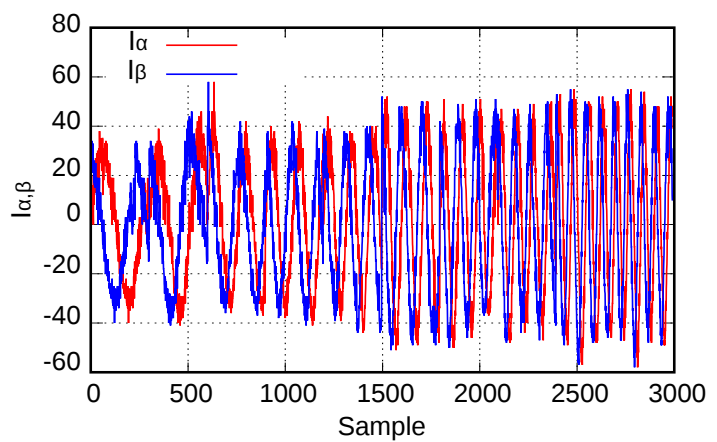

Fig. 13. Experimental $\alpha$ and $\beta$ current data for training.

Such data sets were used as inputs for the training of both neural networks. The desired output data sets are $E_{\alpha}$, for the first network, and $E_{\beta}$, for the second network. Using the acquired position and speed, the back-EMFs were obtained as described in Section III and are shown in Figure 14.

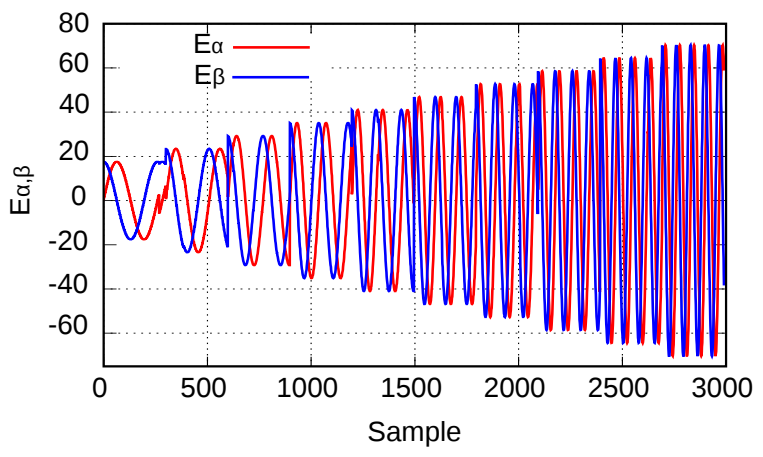

Fig. 14. Experimental $\alpha$ and $\beta$ back-EMF data for training.

\section{B. Execution}

The proposed estimation system was tested experimentally in a set composed by a PMSM with parameters given by Table I, a STM32F103C8T6 microcontroller and an 220V, 200W inverter. The ANNs were trained with floating point variables and exported to $\mathrm{C}$ codes with fixed point variables using multiplications by integer bases. Next, tests without load are firstly presented, followed by tests with the application of load torque steps. The utilized experimental setup is shown in Figure 15.
C. Tests without Load

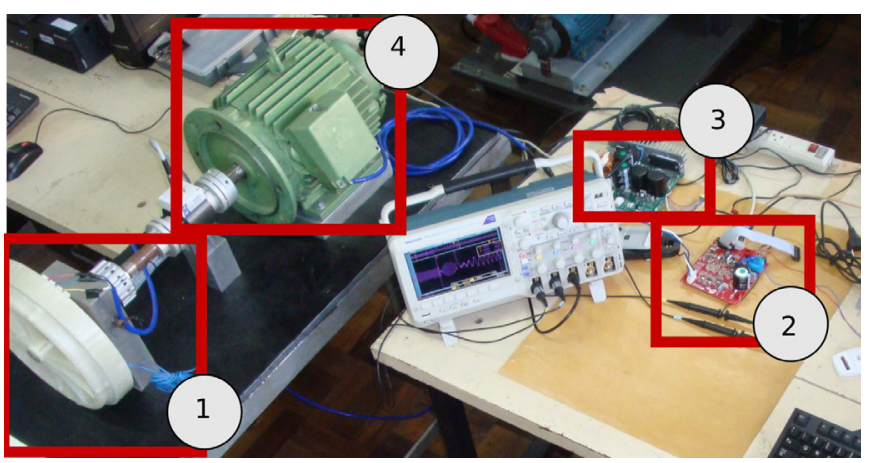

Fig. 15. Experimental setup: (1) PMSM, (2) control, signal acquisition and drive for PMSM, (3) load drive, (4) load.

Figures 16, 17 and 18 show the back-EMF estimations for the motor rotating at 30 RPM, 50 RPM and 100 RPM, respectively. It can be noticed that both the distortion level in the waves amplitudes and harmonic distortions reduce as the rotor speed increases. This is due to the relative noise level in the currents which is reduced as the speed increases.

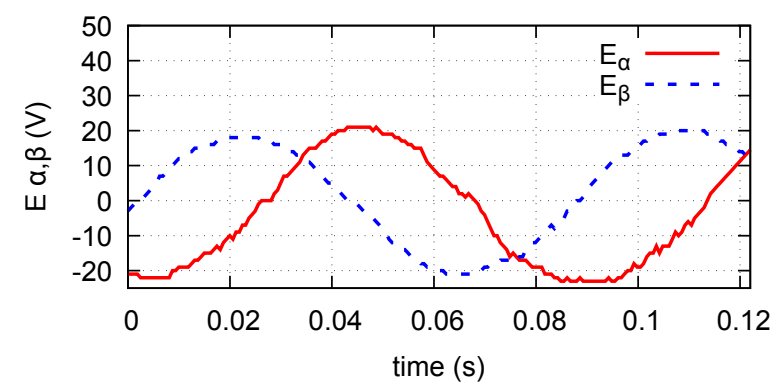

Fig. 16. Back-EMF estimation at 30 RPM.

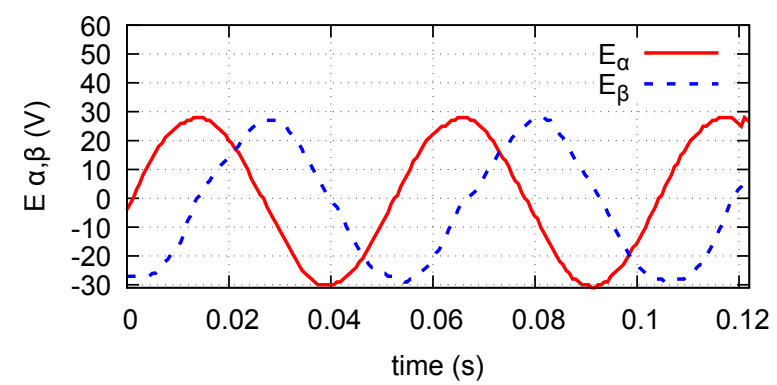

Fig. 17. Back-EMF estimation at 50 RPM.

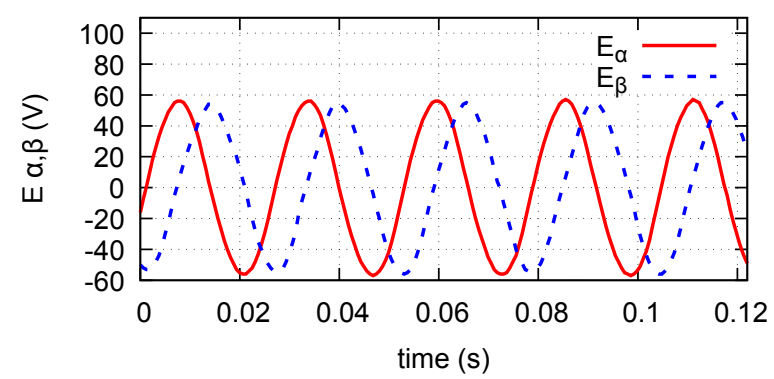

Fig. 18. Back-EMF estimation at 100 RPM. 
Position estimations from the back-EMFs shown in Figures 16, 17 and 18 are depicted in Figures 19, 20 and 21, respectively. It is possible to observe that for a rotation of 100 RPM, the estimation performance is better than in lower speeds, due to the networks inputs noise level.

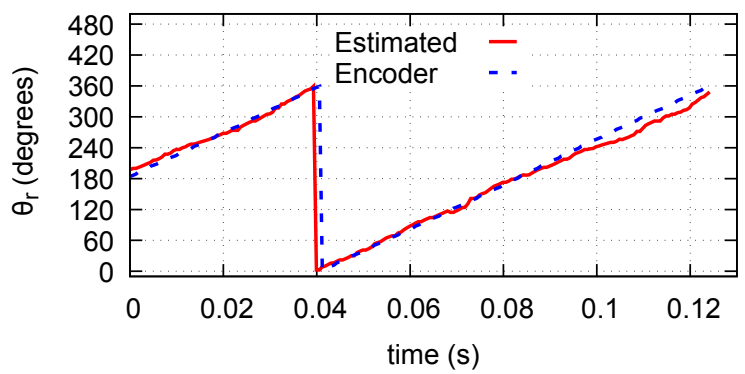

Fig. 19. Position estimation at 30 RPM.

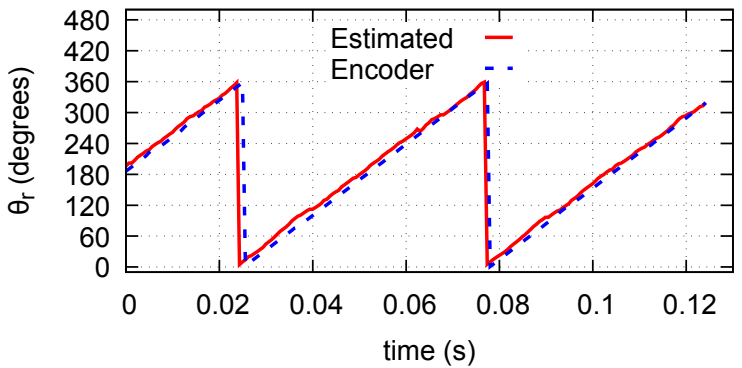

Fig. 20. Position estimation at 50 RPM.

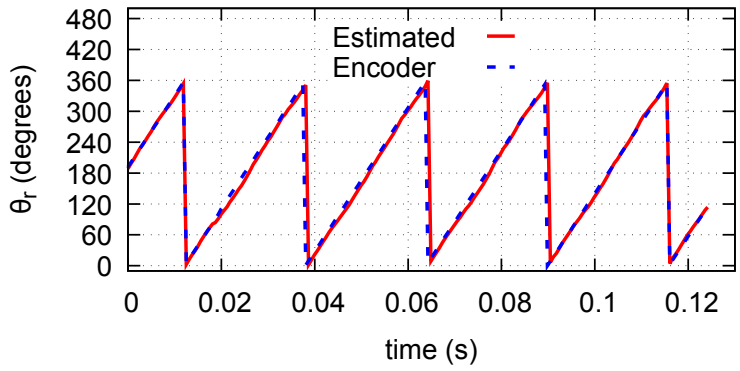

Fig. 21. Position estimation at 100 RPM.

Figure 22 shows the error per sample. Table II shows the absolute mean and the squared mean estimation errors. For both cases, the estimation error is lower at 100 RPM. Considering the mean squared error, the estimator performed better at 50 RPM than at 30 RPM. Considering the absolute squared error, however, the estimator performed better at 30 RPM than at 50 RPM. Roughly speaking, this means that at 50 RPM, the estimation error has smaller peak values than at 30 RPM, which can be interpreted as a more behaved estimation, but with a larger dc error, which can be associated with the training process.

TABLE II

Absolute and Squared Mean Position Estimation Errors

\begin{tabular}{cccc}
\hline Error/Speed & 30 RPM & 50 RPM & 100 RPM \\
\hline Squared & 112.37 & 92.47 & 38.22 \\
\hline Absolute & 7.95 & 8.78 & 4.96 \\
\hline
\end{tabular}

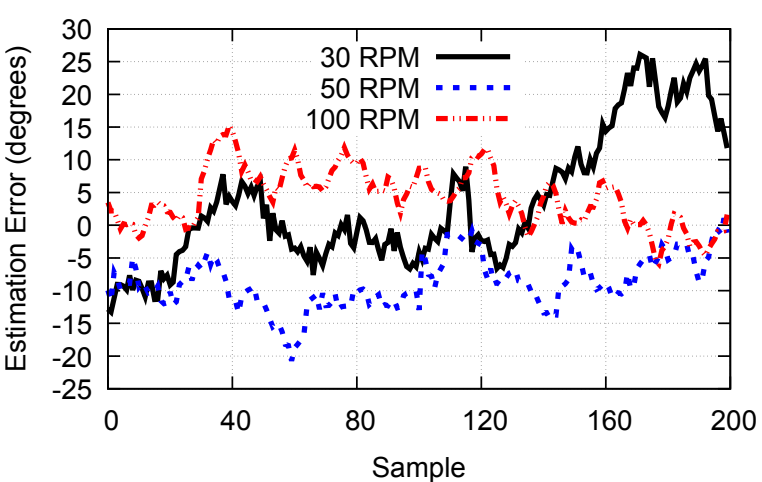

Fig. 22. Position estimation error at 30 RPM, 50 RPM and 100 RPM.

From the estimated position, rotor speed is estimated. A test at speeds of 50 RPM, 100 RPM and 30 RPM is shown in Figure 23. It can be observed that the real speed converges to the reference speeds. The estimated speed, however, presents a high noise level due to derivative calculations, with the occurrence of an offset around 30 RPM.

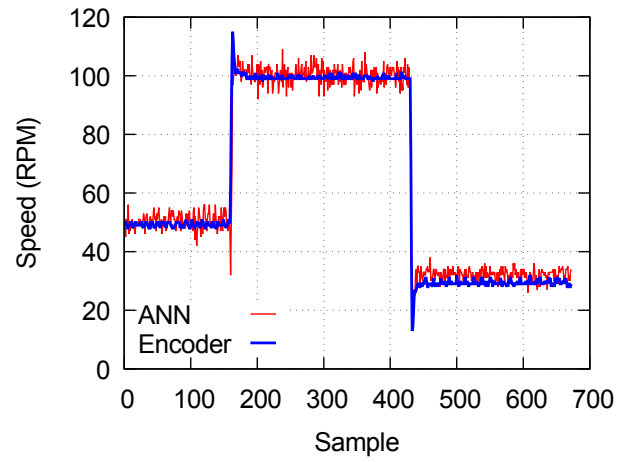

Fig. 23. Rotor speed estimation during reference steps.

\section{Tests with Load}

The load for the following tests were generated by inserting a DC current of approximately $1.5 \mathrm{~A}$ in a three-phase induction machine through two phases, with the third phase blocked, which generates a load torque of approximately $2 \mathrm{Nm}$. The load was inserted in each test by applying a step from a voltage source, after the PMSM reached the reference speed.

Figure 24 presents the measured and estimated positions at a reference speed of 30 RPM. The load torque was inserted at time $t=2.65 \mathrm{~s}$, approximately. In the position curves it can be observed that the motor speed was decreased during the transient caused by the load step, and returns to the initial speed. The estimated position remained close to the measured position even during the load insertion. A similar behavior can be observed in Figure 25, with the application of load at $t=1.82 \mathrm{~s}$ for a 50 RPM reference speed, and Figure 26, with the application of load at $t=2.04 \mathrm{~s}$ for a $100 \mathrm{RPM}$ reference speed. Comparing to the simulation results, it is possible to observe that in the experimental results smaller oscillations were observed. This can be attributed to the load steps being filtered by the electric current transient response of the induction machine, since a step voltage is applied. 


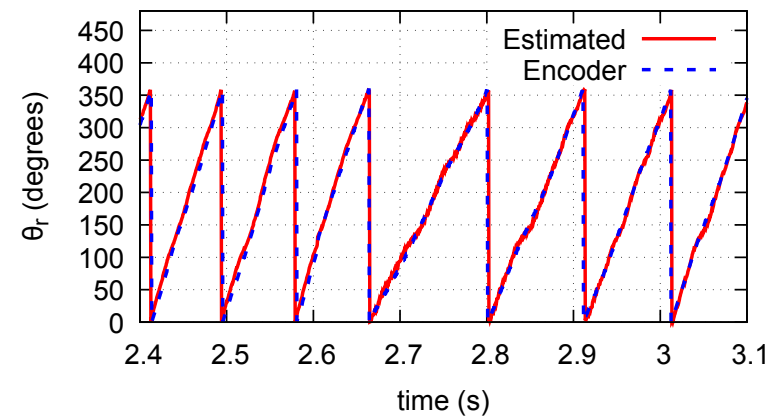

Fig. 24. Position estimation with load at 30 RPM.

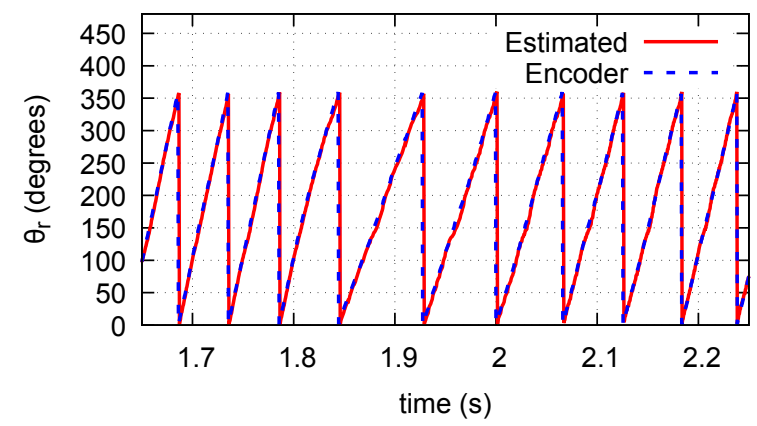

Fig. 25. Position estimation with load at 50 RPM.

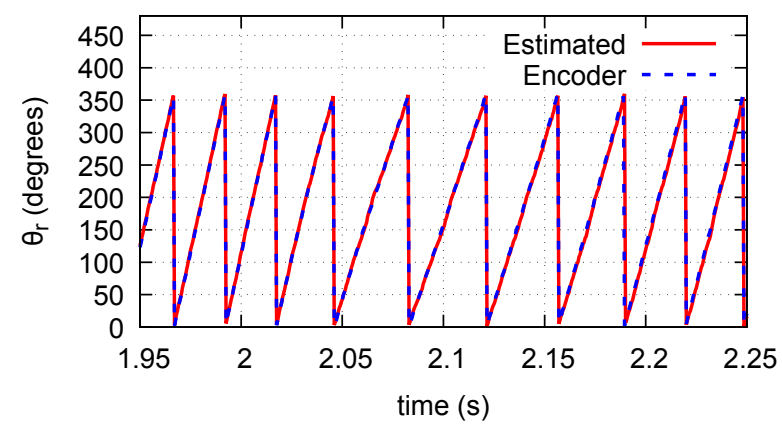

Fig. 26. Position estimation with load at 100 RPM.

\section{CONCLUSION}

An approach for speed and position estimation from back-EMF estimations using FCC-ANNs was presented. Simulation and experimental results are indicatives of the effectiveness of such approach.

It was verified that the training of the networks was simple, performed from a data set taken with varied speed. The networks were training using an open source library and automatically exported to $\mathrm{C}$ codes in the form of functions. The obtained networks are executed in a microcontroller device with suitable computational cost, with satisfactory performance when executed with fixed point variables. Concerning to the quality of the estimated information, there was no clear gain comparing to traditional methods found in literature, such as MRAS and SMO, once the focus of the proposed approach is to simplify the design and implementation of the estimator.

The use of the position estimator has several advantages over the use of a mechanical sensor, such as an encoder.
Among such advantages, the lack of construction and maintenance cost, increase of physical robustness of the drive system and reliability can be mentioned. The mentioned advantages are specifically important in PMSM applications such as in white good appliances, where the reduced volume is a requirement, and in hermetic compressors, in which the motor is drowned in oil, which could compromise the functionality of the mechanical sensor. Simulation and experimental tests could be performed with the use of automatically generated $\mathrm{C}$ code functions, making it practical to embed the trained FCC-ANNs in a fixed point microcontroller. Such approach shows up as an option for position estimation in low cost PMSM drive systems, such as for washing machines applications, with easy tuning and implementation.

\section{ACKNOWLEDGEMENTS}

The authors acknowledge Santa Catarina State University, Brazilian Federal Agency for Support and Evaluation of Graduate Education (CAPES) and EMBRACO.

\section{APPENDIX}

Figure 27 presents excerpts from the generated $\mathrm{C}$ code for $E_{\alpha}$. Firstly, the necessary variables are declared as 32 bit integers and the inputs are scaled. The propagation starts with applying the weighted inputs to all neurons. From the second layer on, the activation function value is calculated and the neuron outputs are propagated forward. The numeric values are the synaptic weights generated from training.

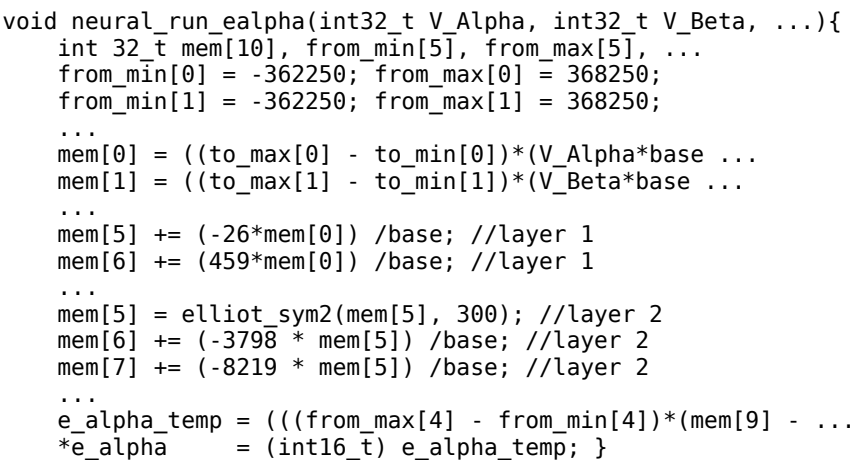

Fig. 27. Excerpts from generated code. The “...” symbol stands for omitted code.

\section{REFERENCES}

[1] F. Betin, G.-A. Capolino, D. Casadei, B. Kawkabani, R. I. Bojoi, L. Harnefors, E. Levi, L. Parsa, B. Fahimi, "Trends in Electrical Machines Control: Samples for Classical, Sensorless and Fault-Tolerant Techniques", IEEE Industrial Electronics Magazine, vol. 8, no. 2, pp. 43-55, June 2014.

[2] T. D. Batzel, K. Y. Lee, "An Approach to Sensorless Operation of the Permanent-Magnet Synchronous Motor Using Diagonally Recurrent Neural Networks", IEEE Transactions on Energy Conversion, vol. 18, no. 1, pp. 100-106, February 2003. 
[3] A. G. Bartsch, G. H. Negri, C. R. Scalabrin, M. S. M. Cavalca, A. Nied, J. de Oliveira, "Predictive Control Approach for Permanent Magnet Synchronous Motor Drive", Eletrônica de Potência, vol. 20, no. 4, pp. 395403, November 2015.

[4] M. Pacas, "Sensorless Drives in Industrial Applications: Advanced Control Schemes", IEEE Industrial Electronics Magazine, vol. 5, no. 2, pp. 16-23, June 2011.

[5] J. C. G. Real, E. V. Sánchez, J. G. Gil, "Position and Speed Control of Brushless DC Motors Using Sensorless Techniques and Application Trends", Sensors, vol. 10, no. 7, pp. 6901-6947, July 2010.

[6] S. Wu, Y. Wang, S. Cheng, "Extreme learning machine based wind speed estimation and sensorless control for wind turbine power generation system", Neurocomputing, vol. 102, pp. 163-175, February 2013.

[7] J. M. G. Villalobos, J. R. Resendiz, E. A. R. Araiza, V. H. Mucino, "A review of parameter estimators and controllers for induction motors based on artificial neural networks", Neurocomputing, vol. 118, pp. 87100, October 2013.

[8] B. Karanayil, M. F. Rahman, C. Grantham, "Online Stator and Rotor Resistance Estimation Scheme Using Artificial Neural Networks for Vector Controlled Speed Sensorless Induction Motor Drive", IEEE Transactions on Industrial Electronics, vol. 54, no. 1, pp. 167-176, February 2007.

[9] B. L. G. Costa, B. A. Angélico, A. Goedtel, M. F. Castoldi, C. L. Graciola, "Differential Evolution Applied to DTC Drive for Three-Phase Induction Motors Using an Adaptive State Observer", Journal of Control, Automation and Electrical Systems, vol. 26, no. 4, pp. 403-420, August 2015.

[10] L. J. S. Shanthi, R. Arumugam, Y. K. Taly, "A novel rotor position estimation approach for an $8 / 6$ solid rotor switched reluctance motor", Neural Computing and Applications, vol. 21, pp. 461-468, April 2012.

[11] K. Premkumar, B. V. Manikandan, "Adaptive NeuroFuzzy Inference System based speed controller for brushless DC motor", Neurocomputing, vol. 138, pp. 260-270, August 2014.

[12] M. George, K. P. Basu, A. T. W. Chiat, "Model reference controlled separately excited DC motor", Neural Computing and Applications, vol. 19, pp. 343350, April 2010.

[13] K. Patan, "Neural Network-Based Model Predictive Control: Fault Tolerance and Stability", IEEE Transactions on Control Systems Technology, vol. 23, no. 3, pp. 1147-1155, May 2015.

[14] M. H. Terra, M. Bergerman, R. Tinós, A. A. G. Siqueira, "Controle Tolerante a Falhas de Robôs Manipuladores", SBA Controle e Automação, vol. 12, no. 2, pp. 73-92, August 2001.

[15] E. R. Marcelo G. Villalva, "Neural Networks Applied to the Control of a Four-Wire Shunt Active Power Filter", Eletrônica de Potência, vol. 10, no. 1, pp. 2330, June 2005.
[16] C. Olivieri, M. Tursini, "A novel PLL scheme for a sensorless PMSM drive overcoming common speed reversal problems", in International Symposium on Power Electronics, Electrical Drives, Automation and Motion, pp. 1051-1056, 2012.

[17] B. M. Wilamowski, "Neural Network Architectures and Learning Algorithms", IEEE Industrial Electronics Magazine, vol. 3, no. 4, pp. 56-63, December 2009.

[18] P. C. Krause, R. R. Nucera, R. J. Krefta, O. Wasynczuk, "Analysis of a Permanent Magnet Synchronous Machine Supplied from a $180^{\circ}$ Inverter With Phase Control", IEEE Transactions on Energy Conversion, vol. EC-2, no. 3, pp. 423-431, September 1987.

[19] D. Grenier, J.-P. Louis, "Use of an extension of the Park's transformation to determine control laws applied to a non-sinusoidal permanent magnet synchronous motor", in Fifth European Conference on Power Electronics and Applications, pp. 32-37, 1993.

[20] E. Sperb, L. Negri, A. Baasch, H. Polli, J. de Oliveira, A. Nied, "Sensorless control of PMSM using a new efficient neural network speed estimator", in International Conference on Power Engineering, Energy and Electrical Drives (POWERENG), pp. 1-5, 2011.

[21] L. H. Negri, "SuperNN", 2014. [Online]. Available at: https://bitbucket.org/lucashnegri/supernn.

\section{BIOGRAPHIES}

Gabriel Hermann Negri, born in March 12, 1992, in Joinville-SC, is an Electrical Engineer (2015) and Master (2016) with Santa Catarina State University. His areas of interest are predictive control, robotics and machine learning.

Filipe Guolo Nazário, born on October 20, 1982, in Criciúma-SC, is an Electrical Engineer (2008), Master (2014) and is currently working at Embraco, developing controls for hermetic compressor refrigeration industry. His areas of interest are electrical machines, sensorless motor control solutions and embedded software development.

José de Oliveira, born in 06/15/1961 in Mandaguari-PR, is an Electrical Engineer (1986), Master (1994) and Doctor in Electrical Engineering (2000) with the Federal University of Santa Catarina. He is currently an associate professor with the Department of Electrical Engineering, Santa Catarina State University. His areas of interest are control systems, electrical machine actuation and power electronics.

Ademir Nied, born in 06/12/1962 in Santo Ângelo-RS, is an Electrical Engineer (1987), Master (1995) and Doctor in Electrical Engineering (2007) with Federal University of Minas Gerais. He is currently an associate professor with the Department of Electrical Engineering, Santa Catarina State University. His areas of interest are electrical machines, control of electrical drives and neural networks. 